\title{
Mejora de la calidad de vida de los mayores a través del Tai Chi y Chi Kung Quality of live improving in elderly people through Tai Chi and Qigong
}

\author{
*Jesús Carrillo Vigueras, **Manuel Gómez Lópezy **Gregorio Vicente Nicolás \\ *Hospital General Universitario Reina Sofia Murcia (España), **Universidad de Murcia (España)
}

Resumen: El continuo crecimiento de la población mayor junto al mantenimiento de la calidad de vida, hacen de la actividad física un aspecto fundamental en el tratamiento integral de los efectos negativos que el paso de los años tiene en nuestra salud, aumentando la esperanza de vida y sobre todo mejorando la calidad de la misma. El Tai Chi y el Chi Kung son modalidades de actividad física muy recomendadas para este grupo poblacional, debido a sus características, a su bajo impacto, posibilidad de practicarlo sin necesidad de instalaciones, materiales o equipamientos especiales, pudiendo practicarse en lugares al aire libre (plazas, parques y jardines), lo que añade igualmente un disfrute del medio ambiente a través del contacto con la naturaleza. Todo esto la hace una práctica ideal para el fomento y promoción de la actividad física en los mayores.

Palabra clave: Salud, Tai Chi, Chi Kung, mayores, actividad física.

Abstract: The continuous growth of elderly people and the high standards of their quality of life, makes the physical activity an important element in the integral treatment of the age negative effects, in order to length the life expectancy and improve the quality of it. The Tai Chi and the Qigong are modalities of physical activity very suitable for this population group, due to their characteristics, to his low impact, not needing facilities, nor material or special equipment for their practice, being able to practice in outdoors spaces (squares, parks and gardens), which also entails to enjoy environment and the nature contact. All this makes an ideal practice for the encouragement and promotion of the physical activity in elderly people. Key words: Health, Tai Chi, Qigong, elderly people, physical activity.

\section{Introducción}

Actualmente, en la sociedad posmoderna en la que nos encontramos, la mejora de la calidad de vida está provocando un aumento de las expectativas de vida de sus ciudadanos (Lópezy Rebollo, 2002; Pastor, 2005), aunque esta, no siempre viene acompañada de una buena calidad de la misma (Serra y Bagur, 2004). El concepto de calidad de vida en los mayores, viene definido por el bienestar físico, las relaciones interpersonales, el propio desarrollo personal, las actividades espirituales y las actividades recreativas tanto activas como pasivas necesarias para mantener la socialización.

Debemos considerar el envejecimiento como un proceso dinámico e individual donde influyen diversos factores que pueden llegar a acelerarlo o retardarlo, como son el estilo de vida, el género, las condiciones ambientales, sociales y económicas (Ticó, 1995). Como afirma Pastor (2005), es muy posible que el ocio sea el aspecto que mejor caracterice a este sector de población mayor de las sociedades postindustriales y la actividad fisica una de las formas de ocupación de ese tiempo de ocio, convirtiéndose esta, en un aspecto fundamental en el tratamiento integral de los efectos negativos que el paso de los años tiene en la salud, aumentando la esperanza de vida y sobre todo mejorando la calidad de la misma (Méndez y Fernández, 2005).

Aunque el deporte aún es una actividad poco frecuente entre los mayores, su práctica regular con la creciente oferta de salud y mantenimiento parece ir en aumento en los últimos años en nuestro país (Otero, 2004; García, 2006), teniendo en cuenta que aunque la prevalencia de inactividad física a cualquier edad es causa de preocupación en la mayoría de los países, es evidente que existe un nivel inferior de actividad física en los grupos de población de mayor edad, siendo incluso menor este nivel en las mujeres (USDHHS, 1996; Armstrong, Bauman y Davies, 2000), y que incluso aun los que son activos, no practican una actividad física suficiente como para obtener beneficios para la salud (USDHHS, 1996; Armstrong, Bauman y Davies, 2000; Taylor et al., 2004). El informe del Surgeon General de los Estados Unidos recoge la recomendación de que un adulto debería dedicar al menos treinta minutos a una actividad fisica de intensidad moderada durante la mayoría de los días de la semana (USDHHS, 1996, 1999), adquiriendo así un

Fecha recepción: 19-05-09 - Fecha envío revisores: 19-05-09 - Fecha de aceptación: 13-06-09 Correspondencia: Jesús Carrillo Vigueras

C/ Rosalía de Castro, 3.

30.120 - Guadalupe (Murcia)

E-mail: jcvigueras@gmail.es comportamiento positivo que favorezca la adherencia a la actividad física (Cauley et al., 2003).

Por lo tanto y a tenor de los resultados, estamos ante un segmento poblacional que es potencialmente, uno de los más permeables a ocupar una buena parte de su tiempo libre con prácticas de ocio activo y saludable como es la actividad físico-deportiva, adecuadas siempre a sus propias condiciones (Espín, 1995; Lehr, 1999).

La relación entre ejercicio fisico y salud, ha sido objeto de innumerables estudios epidemiológicos que coinciden en aseverar que el ejercicio físico es un elemento condicionante de la calidad de vida (Biddle, 1993), de la salud y del bienestar (Blasco, 1994; Corbin, Pangrazi y Welk, 1994), que produce efectos beneficiosos tanto físicos como psicológicos en los mayores (Bennett, Carmack y Gardner, 1982; Matsudo y Matsudo, 1992; Biddle, 1993; Torrado, Aparici y Sanz, 1994;ACSM, 1998; De Gracia y Marcó, 2000; Matsudo, Matsudo y Neto, 2000; Barriopedro, Eraña y Mallol, 2001; Eriksen, 2001; Casterad, Serra y Beltrán, 2003; Myers et al., 2004; Taylor et al., 2004) asociándose a un menor riesgo de mortalidad y morbilidad. Los estudios sugieren que las personas físicamente activas suelen tener dos o tres años más de expectativas de vida (Paffenbarger y Lee, 1996).

$\mathrm{Al}$ igual que en los adultos más jóvenes, aparte de poder obtener beneficios a través de la práctica física a relativamente corto plazo mediante programas de entrenamiento, también se podrán alcanzar muchos beneficios adicionales con una participación prolongada y regular en actividades físicas que fomentan la salud y forman parte de un estilo de vida activo y sano (Bird, 2008). La actividad física regular de intensidad moderada se recomienda en personas de cualquier edad, incluyendo mayores (WHO, 1997; ACSM, 1998). EL ACSM (1990) definió unos niveles de entrenamiento para mejorar la capacidad física de los mayores sanos que consistían en la realización de ejercicios físicos fundamentalmente aeróbicos, donde se produzca la movilización de grandes grupos musculares, durante 20-60 minutos, entre 3-5 días a la semana, haciendo las adaptaciones necesarias, según la diversidad de cada persona. El fundamento para promocionar ejercicios aeróbicos de intensidad moderada en personas mayores se basa en los indicios de su eficacia para reducir el riesgo de enfermedad y promocionar el fitness cardiovascular, que es un componente clave en la determinación de la capacidad funcional de la persona (Bird, 2008) que le permitirá la realización de muchas actividades de la vida diaria, que podrían estar limitadas.

Por ello, de acuerdo con los problemas mencionados anteriormente, por la previsión de un crecimiento del número de personas mayores dependientes en las poblaciones de muchos países, se están desarrollan- 
do numerosas iniciativas de salud pública que promocionan la práctica de actividades físicas, intentado reducir el riesgo y la carga de enfermedades, mejorando el impacto de las patologías existentes y descendiendo la morbilidad provocada por la inactividad (Bird, 2008). Para que estas iniciativas tengan éxito en el estilo de vida de los mayores, es necesario conocer las barreras y los incentivos de la actividad física, ya que son los problemas de salud y la falta de tiempo, las principales barreras a la hora de ser activos (Booth, Bauman y Owen, 2002; Bourdeaudhuij y Sallis, 2002; Schutzer y Graves, 2004), por lo que los programas de ejercicio físico deben ajustarse al estilo de vida y a los valores y obligaciones específicas de la edad.

Nosotros abogamos por la práctica regular de Tai Chi, ya que son muchos los estudios que han demostrado que la práctica de esta actividad en personas mayores influye positivamente en su salud general, ya que es una actividad física que pueden realizarse a lo largo de toda la vida (Portillo, 2002) frenando el proceso de envejecimiento. La práctica regular de Tai Chi tiene un importante efecto sobre muchos tejidos y sistemas, prueba de ello son los resultados encontrados por Choi, Moon y Song (2005), en los cuales queda demostrado que en el sistemaósteomuscular se produce un aumento de la masa muscular y por lo tanto de la fuerza y densidad ósea (Chan et al., 2004), gracias a que esta modalidad física se basa en ejercicios de bajo impacto.

En cuanto al aparato respiratorio se ha demostrado empíricamente que los practicantes de Tai Chi tienen una capacidad respiratoria superior a los de otras modalidades físico-deportivas, mejorando así de manera conjunta el sistema cardiovascular (Taylor-Piliae y Froelicher, 2004). Estas mismas autoras sostienen que gracias a la ejecución de los movimientos se produce un aumento del bienestar y de la salud menta y emocional. De igual forma, la visión también mejora al desarrollar una mayor amplitud en el campo visual debido al trabajo que se efectúa tanto en la conciencia de esa visión como en la ejercitación de la musculatura del ojo (Carrillo, 2008).

Otros elementos que mejoran sustancialmente y que son determinantes en el día a día de los mayores es la flexibilidad (Zhang et al., 2006) y el equilibrio (Tsang et al., 2004; Tsang y Hui-Chan, 2003, 2004a y b; Taylor-Piliae et al., 2006), que junto con el aumento de la fuerza muscular y la densidad ósea anteriormente mencionadas, previenen de las caídas, tan habituales a estas edades.

Estudios como el realizado por Chwan-Li et al. (2007), demuestran de forma inequívoca que mediante esta modalidad de actividad física, se actúa sobre el sistema endocrino, no solo sobre el eje hipotálamohipofisario-adrenérgico, demostrado sobradamente, sino también sobre el metabólico, responsable del control de la glucosa en las personas que tienen algún déficit de control por la circunstancia que fuese.

Finalmente, a nivel psicológico, los practicantes de Tai Chi experimentan mayor seguridad -como resultado de todos los beneficios descritos anteriormente-, una mejora de la memoria, gracias al aumento del riego sanguíneo y a los distintos movimientos que la persona tiene que memorizar y una disminución del estrés, provocando al mismo tiempo un descenso de la tensión arterial, la cual se encuentra normalmente aumentada en estas edades. A todo lo dicho hasta ahora, hay que añadirle que la práctica regular de ejercicio aeróbico, hace que estas personas puedan conciliar mejor el sueño, recuperándose antes y mejor del esfuerzo físico, ya que «Dondefluye la sangrefluye la vida y donde no hay riego nada vive» (Carrillo, 2008).

\section{Implicaciones metodológicas}

Todo programa de actividad física debe ir más allá de los beneficios biológicos, ya que tenemos que conseguir la consolidación, estabilidad en el tiempo y adherencia al mismo, considerando a los practicantes, los objetivos a conseguir, contenidos y una adecuada metodología de trabajo (Ticó, 1995). Este programa de ejercicio físico, el cual puede ir orientado al antienvejecimiento en cualquiera de las dimensiones humanas, debe ser intencional y estar compuesto tanto por una variedad de tareas motrices que logren estimular los distintos procesos psicológicos, como por una adecuada organización que favorezca la consecución de los objetivos marcados, los cuales no deben ser complicados o alcanzables a largo plazo.

Debido a que no todos los mayores pueden someterse al mismo tipo de actividad física y que la evaluación de la actividad física en el mayor plantea problemas a causa de que la mayoría de los métodos utilizados han sido concebidos para los jóvenes (Lafont et al., 1997), antes de comenzar el programa de ejercicio físico, es aconsejable realizar una evaluación previa con el fin de evitar determinados movimientos en algunas patologías cervicales. Para ello intentaremos exigir un certificado médico y utilizaremos un cuestionario rápido y fiable que evalúe el estilo de vida de la persona (Heyward, 2006).

Igualmente hay que resaltar que no existen actividades específicas para los mayores, sino que lo más importante es que estén adaptadas a sus posibilidades y características, se presenten de manera adecuada, ocupen en la programación el lugar oportuno y reciban un tratamiento didáctico que permitan a los participantes practicarla con éxito (Camiña, Cancela y Romo, 2001).

Teniendo estas premisas en mente, no debemos elevar la competencia motriz de estas personas mediante el establecimiento de tareas en las que los ritmos son impuestos externamente, reclamando para ello exigencias demasiado elevadas ya que haremos que se sienta no competente y con falta de seguridad, generándole estrés y ansiedad (Ruiz, 1995).

Debemos incentivar ejercicios dinámicos que impliquen la movilización de grandes masas musculares, cíclicos o rítmicos, evitando aquellas actividades que pueden llegar a provocar aumentos importantes de la presión arterial (Meléndez, 2000).

Las sesiones se llevarán a cabo con un máximo de veinte participantes, varios días a la semana con una duración de unos sesenta minutos aproximadamente en principio, para posteriormente ir alargando las clases en función de la mejora de la condición física del grupo. Un aspecto importante es que durante la práctica haya música de fondo relajante que incite a movimientos lentos y pausados, estando esta estructurada en un calentamiento de articulaciones y músculos de unos diez a doce minutos, seguido del cuerpo principal de la actividad que tendrá una duración de unos 40 minutos aproximadamente, para finalizar con una vuelta a la calma que puede comprender ejercicios de relajación en posición de acostado, sedentación o incluso en movimiento o bien, con ejercicios de auto-masaje o masaje por parejas, utilizando para ello materiales como pelotas de goma, pesas, etc.

\section{Propuesta práctica de tai chi y chi kung para personas} mayores

El Tai Chi tiene distintas corrientes entre la que destacan la marcial, que se practica principalmente en China (Wu Shu) y la terapéutica, entre otras, que se realiza como un arte marcial interno, donde los movimientos son más lentos, pausados, con mayor armonía (Chau y Mao, 2006) y se practica en casi todos los continentes. El apelativo de interno se le da a las artes de guerra (marciales) que no persiguen la lucha contra un contrincante, sino que por el contrario persiguen la perfección en el movimiento y el obtener un beneficio terapéutico a través del movimiento en sí.

Los movimientos de Tai Chi se realizan por medio de una secuencia estandarizada mediante desplazamientos donde la pelvis y la columna son un eje básico del movimiento. Si bien el Tai Chi es la técnica más conocida, existen otras no tan conocidas que se pueden adaptar mucho mejor para iniciar una sesión o bien iniciar a un grupo de principiantes. Si contamos además con determinados problemas de salud relacionados con la memoria observaremos que la elección de movimientos más sencillos es primordial para asegurar una buena adherencia a la actividad fisica en personas mayores.

La técnica más usada en las sesiones es el Chi Kung o Qi Gong, ambas significan lo mismo. Podríamos definir el Chi Kung (Qi Gong) como una gimnasia china, suave, lenta, y armoniosa, que nos permite 


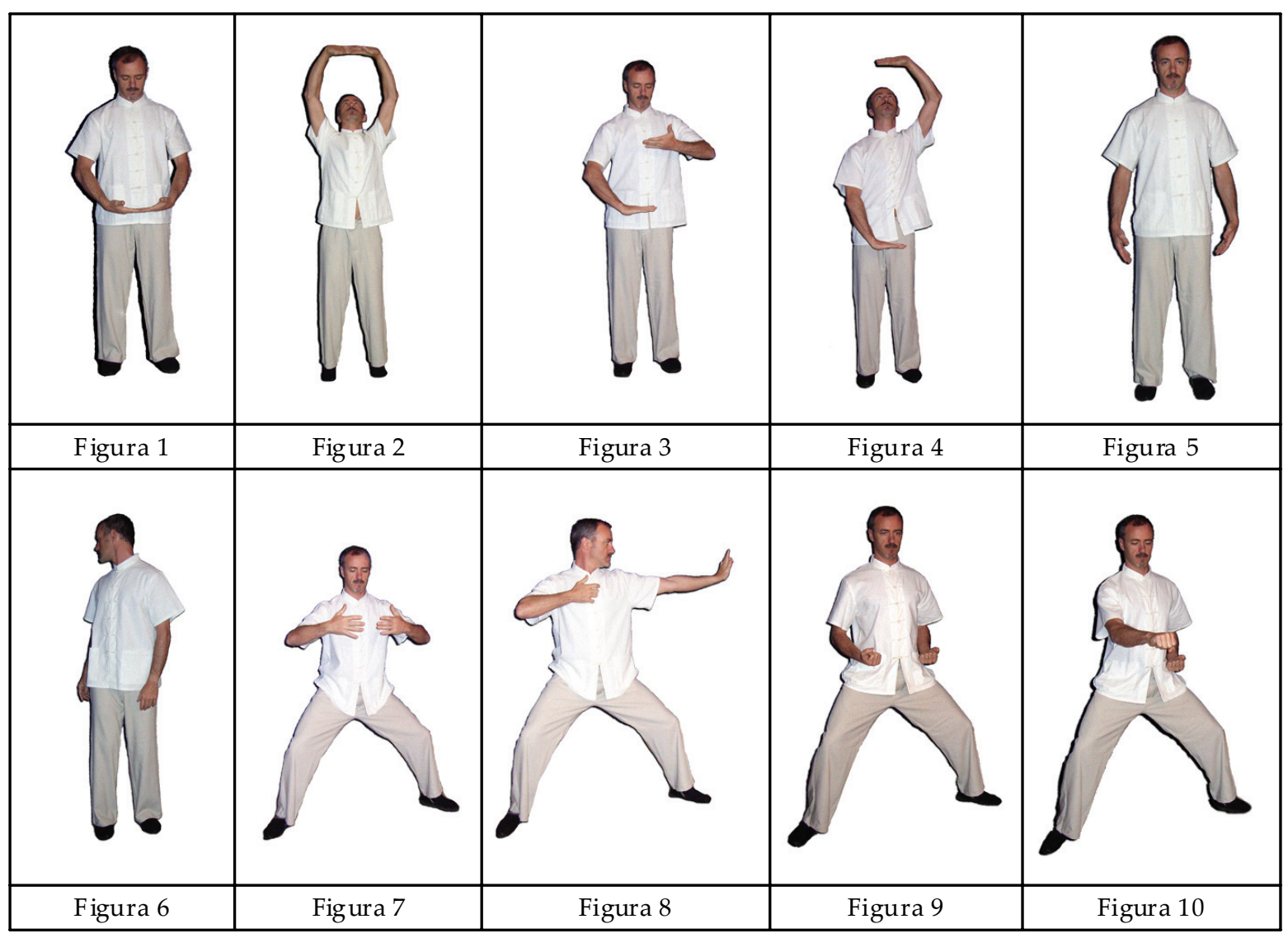

desarrollar totalmente nuestro potencial con el fin de mantener la salud. Con frecuencia, cuando a una persona se le aconseja que practique Yoga o Tai Chi, realmente lo que se le está proponiendo es que realice sencillos ejercicios de Chi Kung, puesto que las primeras técnicas no serían recomendadas en muchos de los casos.

La práctica del Chi Kung es recomendada como técnica de iniciación para personas sedentarias, debido a que puede realizarse en un espacio reducido, tan pequeño como el lugar que ocupa una colchoneta, mientras que por el contrario en el Tai Chi se requiere de un espacio amplio y al aire libre.

Los ejercicios contemplan un aspecto interno y otro externo. El aspecto interno busca mejorar la respiración mediante ejercicios estáticos o dinámicos y, como consecuencia de ésta, tranquilizar la mente y tonificar la energía corporal con ayuda del aire y los alimentos. En el aspecto externo, se busca fortalecer la estructura muscular, mantener fuertes y flexibles los tendones, mejorar la movilidad articular evitando enfermedades óseas y estimular los sentidos.

A continuación se describe brevemente los diferentes movimientos que componen la propuesta práctica de Ba Duan Jin ó Pa Duan Jing (Las ocho piezas del brocado de seda ó Las ocho joyas del Tai Chi).

Existen muchas variaciones de los ocho ejercicios, en especial de la forma de pie que es la más popular. Entender la intención y finalidad de los mismos, su origen y su propósito hará comprender cualquiera de sus variantes y su ejecución será más efectiva. La práctica de los ejercicios requiere relajación, suavidad y cierta perfección. La respiración será ligera y acompañará a los movimientos. Las variaciones practicadas deben ser acordes a nuestra capacidad y al nivel del practicante, teniendo en cuenta que puede ser necesario mucho tiempo para adquirir un dominio total de la técnica. Según nuestro nivel u objetivos insistiremos en unos u otros aspectos de este arte: la parte gimnástica, la respiración, la relajación, la meditativa, etc.

Las sesiones se deben de realizar atendiendo a los siguientes parámetros (Chwan-Li Shen, Du Feng et al, 2007): sesiones de una hora de duración y de periodicidad bisemanal, los mejores resultados se obtienen a partir de las 12 sesiones. Las sesiones se realizarán con una música de fondo que permita la relajación en los movimientos y la focalización en la respiración, sobre todo al principio.

Los nombres de las ocho piezas son:

1. Dos manos sujetan el cielo para armonizar el Triple Recalentador.

2. Estirar una mano hacia los pies y después la otra para armonizar el bazo y el Estómago.

3. Girar la cabeza y mirar hacia atrás para evitar consumirse.

4. Abrir el arco a izquierda y a derecha para dispararle al halcón.

5. Baja el cuerpo y aprieta el puño con ojo de enfado.

6. Empinarse en la punta de los pies y rebotar siete veces.

7. Menea tu cola y balancea tu cabeza para liberar el fuego de tu corazón.

8. Llevar las manos hacia el suelo y agarrarse los pies.

Primera pieza: dos manos sujetan el cielo para armonizar el Triple Recalentador.

Colocar el cuerpo en la posición de Wu Chi: de pie con los pies separados el ancho de las caderas, la pelvis ligeramente basculada hacia adelante(figura 5).

Flexionar ligeramente las rodillas, con la espalda recta y sin sacar las nalgas. Entrecruzar los dedos o enfrentar los dedos por la punta de estos delante del abdomen con las palmas mirando hacia arriba (figura 1).

Elevando al mismo tiempo las manos y el cuerpo, la respiración desciende hasta el abdomen, los pulmones se llenan de aire mientras que ascendemos. Al llegar a la cara, las palmas giran hacia nosotros para orientarse hacia arriba.

Seguir levantando las palmas al tiempo que se despegan los talones del suelo. Finalmente subir los brazos totalmente extendidos, mientras hundimos las puntas de los pies en el suelo levantando los talones (figura 2).

Al elevar las manos, se ha de tener en cuenta que lleguen a quedar casi en ángulo recto en relación con los brazos y que éstos estén lo más rectos posibles por encima de la cabeza. Relajar los músculos de los 
hombros y cuello. La pelvis ha de estar basculada y la espalda recta. Los ojos siguen a las manos en todo momento trabajando de forma sutil el campo visual.

Segunda pieza: estirar una mano hacia lo alto y la otra hacia los pies para armonizar el bazo y el estómago.

Partir de la posición inicial, Wu Chi (figura 5). Situar las manos paralelas, a la altura del estómago frente al Tan Tien (punto de equilibrio corporal situado a $3 \mathrm{~cm}$ por debajo del ombligo y $3 \mathrm{~cm}$ hacia adentro), las palmas enfrentadas, es decir, una mano hacia arriba y a la misma altura la otra mano hacia abajo (empezar a inhalar) (figura 3).

Separar las manos, una sube por encima de la cabeza con la palma hacia arriba, los dedos apuntando en la dirección del hombro opuesto; la otra desciende con la palma hacia abajo, hasta dejar el brazo al costado y la palma de la mano mirando hacia el suelo. Elevar la mirada hacia la mano que está encima de la cabeza (figura 4).

Llevar las manos de nuevo a la altura del ombligo exhalando y haciendo un pequeño arco por delante, pero invirtiendo la postura del comienzo para separar las manos una vez más pero alternando los brazos.

Hay que tener la sensación de que las manos empujan algo que ofrece cierta resistencia, pero sin poner los músculos en tensión excesiva. La respiración nunca debe ser forzada, por lo que la velocidad del ejercicio dependerá de la respiración y no al contrario. Se va a desarrollar de esta forma una respiración completa, abdominal y torácica. También efectúa un estiramiento de los tejidos que sujetan a los órganos internos que reciben un masaje suave y de los tendones. Se ha de sentir cómo se liberan los posibles bloqueos producidos por la tensión acumulada gracias a la acción integral de todos los tejidos, huesos, tendones, músculos y órganos.

Tercera pieza: girar la cabeza y mirar hacia atrás para evitar consumirse.

Partir desde la posición inicial, Wu Chi, (figura 5) a la que se ha regresado después del ejercicio anterior. (inhalar) Sin girar la cintura ni el pecho volvemos la cabeza a la izquierda cuanto nos sea posible exhalan- do en este movimiento; luego, cuando hayamos alcanzado el límite de la rotación cervical, la prolongamos con el raquis girando el hombro izquierdo hacia atrás, pero sin que la pelvis acompañe el movimiento. Acabamos con una ligera rotación de las caderas a la izquierda para permitir que la vista se dirija hacia atrás (figura 6). A este ejercicio se le atribuye un efecto de masaje del intestino grueso debido a que el movimiento nace del coxis y se trasmite por el cinturón abdominal a toda la espina hasta que se libera con la cabeza. Es una de las piezas que tiene más dificultad por involucrar un movimiento interno. La cabeza sólo debería girarse como resultado de este movimiento interno y en ningún caso se forzarán las vértebras cervicales. Los principiantes deberían tener precaución con la cabeza al dirigir la mirada al talón. Este movimiento puede limitarse a bajar la mirada sin mover el cuello. La cabeza ha girado sobre un eje perfectamente vertical; la mirada se dirige en horizontal lejos, y describe un ángulo exacto de $180^{\circ}$.

Inhalar retornando lentamente a la postura inicial, la mirada siempre acompañando de frente al movimiento que se realizará rectificando primero la pelvis, luego los hombros y, por último, el cuello.

La variación más común en China de este ejercicio consiste en llevar brazos por delante a la altura del Tan Tien y girar levemente la cabeza y los hombros, siempre sin llegar a mover la cadera.

Cuarta pieza: abrir el arco a izquierda y a derecha para dispararle alhalcón

Doblar los brazos hacia el cuerpo a la altura del pecho inhalando. Damos un paso a la izquierda y doblamos las rodillas para adoptar la posición de un jinete (figura 7).

Girar el torso hacia la izquierda. Extendemos los dedos índice y pulgar de la mano izquierda y cerramos los otros dedos. Figura 7

Empujar hacia la izquierda con la mano del mismo lado y levantamos el codo contrario hacia la derecha; mantenemos los ojos fijos en la mano izquierda y el codo derecho a la altura del hombro. La mano derecha tensa el arco y la mirada apunta a través del índice a lo lejos. En la mano izquierda estiramos el índice y el pulgar. Esta acción se puede realizar con el resto de dedos plegados o estirados (figura 8).

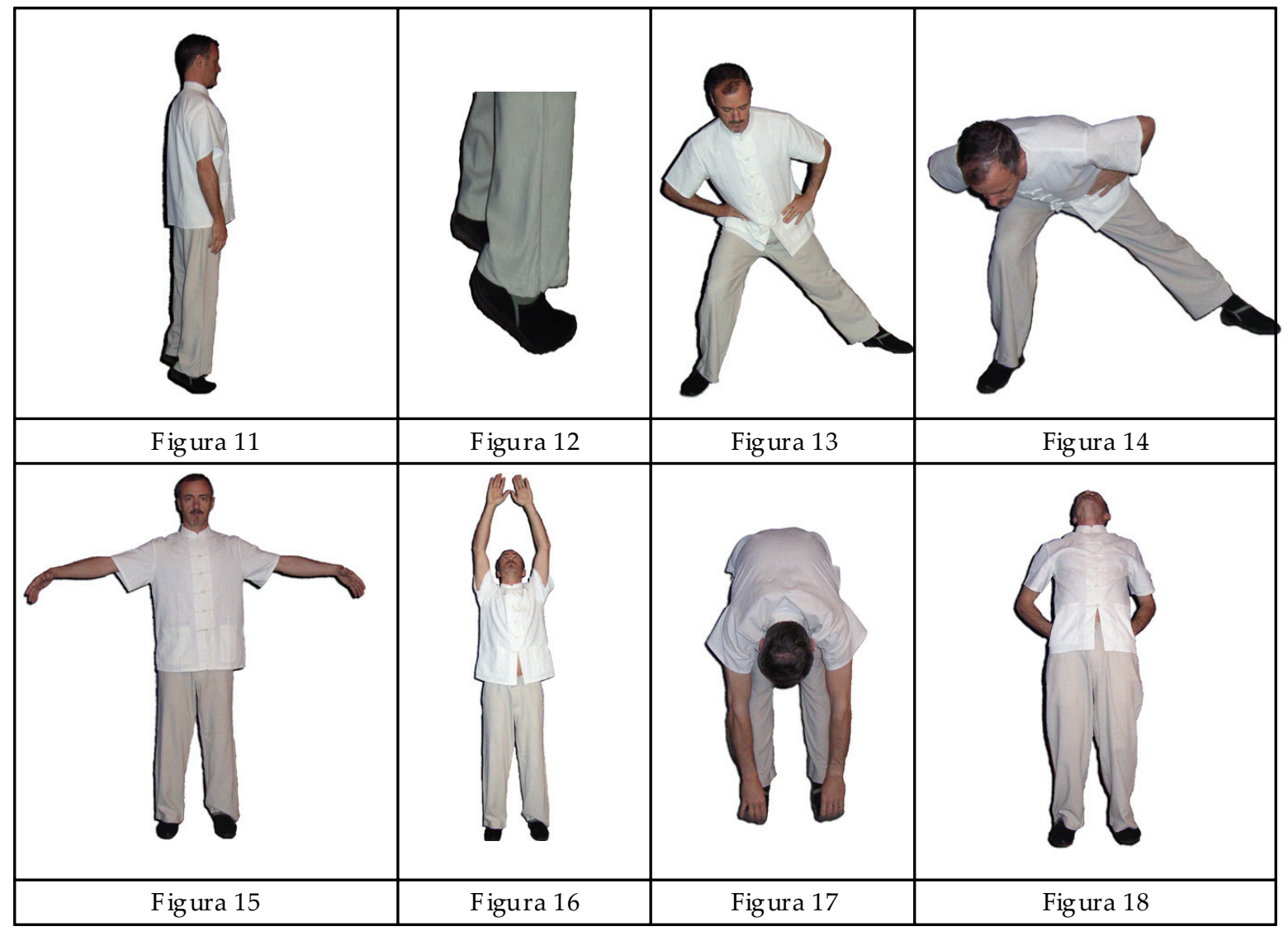


Mientras desplegamos los brazos el pecho, va girando hacia el frente de forma que al final estamos con la mirada hacia la izquierda pero con el pecho orientado hacia delante y vamos descendiendo hasta quedar en la posición inicial.

Los beneficios de este movimiento se relacionan con el pulmón por lo que al realizar el ejercicio buscaremos una sensación de apertura y liberación en el pecho.

Quinta pieza: baja el cuerpo y aprieta el puño con ojo de enfado.

Dar un paso a la izquierda y doblar las rodillas para adoptar la posición de un jinete. Mantener erguida la parte superior del cuerpo, con los muslos paralelos al suelo, en la medida de lo posible. La pelvis basculada mantiene recta la columna, sin flexionar las vértebras lumbares. Las rodillas no han de sobrepasar la punta de los pies, de lo contrario se podría producir una lesión.

Doblar los brazos hacia el cuerpo a la altura de la cintura, los puños cerrados con el dorso de la mano hacia abajo y relajados. La vista se dirige hacia el frente, con los ojos bien abiertos (figura 9).

Iniciar el ejercicio inhalando. Posteriormente exhalando se despliega el brazo izquierdo hacia el frente mientras se aprieta el puño con fuerza, el círculo formado por el pulgar y el índice queda mirando hacia lo alto. El puño cerrado y la mirada fiera centrada en un punto imaginario en el horizonte, el puño va girando hacia adentro para colocarse de forma natural con el pulgar abajo. Abrimos la mano al llegar a la extensión máxima con el pulgar hacia abajo, giramos la mano y recogemos el pulgar dentro de resto de dedos. Los músculos de cara, cuello y hombros han de estar relajados. Apretamos con suavidad el entrecejo para estimular el inicio del canal de vesícula biliar y el de vejiga (figura 10).

Relajar la mirada y los puños mientras el brazo regresa a la altura de la cintura inhalando en el acercamiento de la mano hacia el cuerpo. Repetimos el movimiento con el puño derecho.

Sexta pieza: empinarse en la punta de los pies y rebotar siete veces.

Partir de la posición inicial. Mantener bien alineados el cuerpo y la cabeza. Se eleva el cuerpo poco a poco sobre la punta de los pies, inhalando y empujando con la cabeza hacia arriba. El mentón queda recogido hacia el cuello (figura 11)

Descender dejando caer de golpe el talón en el suelo exhalando el aire, con cierta suavidad al principio. Repetir el ejercicio siete veces que es el número de repeticiones considerado como un ciclo (figura 12).

Se puede realizar a diferentes velocidades, si lo hacemos lentamente incrementaremos la fuerza de las piernas y la circulación de retorno, y por tanto, la limpieza de la sangre, además de favorecer el desarrollo del equilibro. Realizado de forma rápida y suelta ayuda a distribuir el chi por todo el cuerpo; en este caso la respiración será libre.

Séptima pieza: menea tu cola y balancea tu cabeza para liberar el fuego de tu corazón.

Dar un paso a la izquierda y doblar las rodillas para adoptar la posición de un jinete. Apoyar las manos en la cintura. Flexionar el tronco hacia delante llevándolo a la horizontal. La espalda ha de mantenerse recta.

Empezar inhalando. El tronco parte de la posición erguida, describe una semicircunferencia bajando desde la pierna derecha hacia la izquierda, al tiempo que se desplaza el peso del cuerpo desde la pierna derecha hasta la pierna izquierda, que permanece flexionada, mientras la derecha se estira (figura 13); bajamos haciendo un círculo con el cuerpo mientras se exhala. Una vez abajo, se vuelve a cambiar el peso a la pierna derecha (figura 14), mientras la pierna izquierda se va estirando. Al llegar a la pierna derecha, empezamos a subir inhalando hasta llegar al centro de nuevo y continuar con el ciclo.

Octava pieza: llevar las manos al suelo y agarrarse los pies.

Partiendo de la posición inicial de Wu Chi nos agachamos ligeramente, con la espalda recta y sin sacar las nalgas (figura 5).

Inhalando elevamos los brazos lateralmente por encima de la cabeza hasta juntar los dedos pulgares (figura 15). Seguimos siempre nuestras manos con los ojos trabajando de esta manera nuestro campo visual. Volvemos las palmas hacia adelante y la espalda se mantiene recta metiendo el coxis (figura 16).
Exhalando las manos, descienden con los brazos extendidos, por delante del cuerpo, hasta agarrar los dedos de los pies (si es necesario doblamos las rodillas) (figura 17), siempre debemos mirar nuestras manos. La espalda recta en la misma línea de brazos y manos. Tirar ligeramente de los dedos de los pies. La espalda está erguida para permitir la circulación de la energía. Inhalando erguimos el cuerpo con la ayuda de los brazos que se levantan por delante y, haciendo un círculo, dejamos reposar las manos en la zona lumbar.

Estiramos el torso ligeramente hacia atrás mientras elevamos la mirada hacia arriba y vaciamos el aire que nos quede en los pulmones con la boca abierta (figura 18).

La repetición de cada uno de los ejercicios se hará dependiendo del estado físico de los practicantes, debiendo, sobre todo al principio hacer pausas para evitar el cansancio en las piernas por lo estático del movimiento. Para evitar esta situación se mezclan estos ejercicios de una forma diferente permitiendo una mayor movilidad del tren inferior.

\section{Conclusiones}

El crecimiento de la población mayor subraya la importancia de mantener la calidad de vida en las personas de avanzada edad (Serra y Bagur, 2004). La disposición de un tiempo libre considerable, unido a un espíritu dinámico, hace que sea un segmento poblacional activo en potencia, siempre y cuando seamos capaces de ofertarles programas de actividades físico-deportivas bien planificadas, organizadas y supervisadas por un especialista que logre adaptar dicha oferta a las características personales de cada uno de ellos, respondiendo a sus expectativas de mantenimiento y/o mejora de la salud, evitando así sentimientos de marginación y falta de integración en la sociedad, y enlenteciendo el deterioro físico y cognitivo causado por el paso de los años. Mediante la práctica regular de Tai Chi y Chi Kung la persona mayor experimentará cambios que pueden afectar a dos niveles:

Por un lado, a corto plazo, la sensación de bienestar que es inmediata al mejorarla respiración. El equilibrio se recupera de forma paulatina y las patologías asociadas al déficit circulatorio como es la hipertensión, las parestesias, la falta de memoria, etc. mejoran de forma notable. Igualmente, la capacidad respiratoria aumenta de manera gradual y los recursos existentes son mayores por lo que demandan más actividad física y más ocio.

Y por último a largo plazo, duermen mejor y son más autónomos, gracias a una mayor sensación de estabilidad provocada por el aumento de fuerza en las extremidades. Resaltar también que gracias a la mejora del equilibrio, de la flexibilidad en las articulaciones y de su densidad ósea, hace que descienda el riesgo de fracturas por caídas. Mentalmente son capaces de centrar mucho mejor sus ideas y de recuperar la memoria perdida ante estímulos nuevos que suponen retos que hacen que las conexiones neuronales se restablezcan.

Es vital que desde la perspectiva de la salud y de su promoción, no olvidemos que la actividad física no será completamente eficaz a no ser que se dirija a los incentivos y a las barreras que influyen en los mayores (Bird, 2008), adaptando igualmente los componentes de intensidad, duración y frecuencia del ejercicio físico a la capacidad y objetivos de cada uno de ellos.

Por lo tanto consideramos al Tai Chi y al Chi Kung como una actividad física muy aconsejable e ideal para el fomento y promoción de la actividad física en los mayores, gracias a sus características de bajo impacto y al no necesitar instalaciones ni materiales o equipamientos especiales para su práctica, ya que puede practicarse en lugares al aire libre como plazas, parques y jardines, lo que conlleva también disfrutar del medio ambiente y del contacto con la naturaleza que adicionalmente tienen un marcado efecto anti-estrés.

\section{Bibliografía}

ACSM (1990). The recommended quantity and quality of exercise for developing and maintaining cardiorespiratory and muscular fitness in healthy adults. Medicine and Science in Sports and Exercise, 22, 265-274. 
ACSM (American College of Sports Medicine) (1998). Position stand: Exercise and physical activity for older adults. Medicine and Science in Sports and Exercise, 30, 992-1008

Armstrong, T., Bauman,A., Davies, J. (2000). Physical activity patterns of Australian adults. Results of the 1999 National Physical Activity Survey. Australian Institute of Health and Welfare, Canberra Australian Bureau of Statistics 2004 Online. Available: http://www.abs.gov.au [Consultado: Noviembre, 2008].

Barriopedro, M.I., Eraña, I. \& Mallol, L. (2001). Relación de la actividad física con la depresión y satisfacción con la vida en la tercera edad. Revista de Psicología del Deporte, 10, 239-246.

Bennett, J., Carmack, M.A. \& Gardner, V.J. (1982). The effect of a program of physical exercise on depression in older adults. Physiology Educational, 39, 21-24.

Biddle, S. (1993). Psychological benefits of exercise and physical activity. Revista de Psicología del Deporte, 4, 99-107.

Bird, S. (2008). Ejercicio y ancianos. En K. Woolf-May (Coord.) Prescripción de ejercicio: fundamentos fisiológicos. Guía para profesionales de la salud, del deporte y del ejercicio fisico (pp. 203-218). Barcelona: Elsevier España.

Blasco, T. (1994). Actividad fisica y salud. Barcelona: Martínez Roca.

Booth, M.L., Bauman, A., \& Owen, N. (2002). Perceived barriers to physical activity among older Australians. Journal of Aging and Physical Activity, 10 , 271-280.

Bourdeaudhuij, I.D., \& Sallis, J. (2002). Relative contribution of psychosocial variables to the explanation of physical activity in three population-based adult samples. Preventive Medicine: And International Journal Devoted to Practice and Theory, 34, 279-288.

Camiña, F., Cancela, J.M. \& Romo, V. (2001). La prescripción del ejercicio físico para personas mayores. Valores normativos de la condición fisica. Revista Internacional de Medicina y Ciencias de la Actividad Física y del Deporte, 2. Online. Available: http://cdeporte.rediris.es/revista/revista2/mayores.htm[Consultado: Noviembre, 2008].

Carrillo, J. (2008). Técnicas de afrontamiento del estrés: Tai Chi. Murcia: SATSE.

Casterad, J.C., Serra, J.R. \& Beltrán, M. (2003). Efectos de un programa de actividad física sobre los parámetros cardiovasculares en una población de la tercera edad. Apunts: Educación fisica y deportes, 73, 42-48.

Cauley, J.A., Stone, K., Thompson, T.J., Bauer, D.C., Cummings, S.R. \& Ensrud, K.E. (2003). Relationship of changes in physical activity and mortality among older women. Journal of the American Medical Association, 289, 2379-2386.

Chan, K., Qin, L., Lau, M., Woo, J., Au, S., Choy, W., Lee, K. \& Lee, S. (2004) Arandomized, prospective study of the effects of Tai Chi Chun exercise on bone mineral density in postmenopausal women. Archives of Physical Medicine and Rehabilitation, 85, 717-722.

Chau, K.W. \& Mao, D.W. (2006). The characteristics of foot movements in Tai Chi Chuan. Research in Sports Medicine, 14, 19-28

Choi, J.H., Moon, J.S. \& Song, R. (2005). Effects of Sun-style Tai Chi exercise on physical fitness and fall prevention in fall-prone older adults. Journal of Advanced Nursing, 51, 150-157.

Chwan-Li Shen, Du Feng, Esperat, M.C.R., Irons, B.K., Ming-C. Chyu, Valdez, G.M. \& Thompson, E.Y.(2007) Effect of Tai Chi Exercise on Type 2 Diabetes:A Feasibility Study. Integrative Medicine Insights, 2 15-23

Corbin, C.B., Pangrazi, R.P. \& Welk, G.J. (1994). Towards an understanding of appropriate physical activity levels for youth. Physical Activity and Fitness Research Digest, 1, 1-8.

De Gracia, M. \& Marcó, M. (2000). Efectos psicológicos de la actividad física en personas mayores. Psicothema, 12, 285-292.

Eriksen, G. (2001). Physical fitness and changes in mortality: the survival of the fittest. Sports Medicine, 31, 571-576.

Espín, M. (1995). Sociología del envejecimiento. En J.F. Marcos Becerro, W Frontera \& R. Santonja Gómez (Coords.) La Salud y la Actividad Física en las Personas Mayores-Tomo I-(pp. 295-301). Madrid: Santonja-Comité Olímpico Español (COE).

García, M. (2006). Posmodernidad y Deporte: entre la individualización y la masificación. Encuesta sobre hábitos deportivos de los españoles 2005. Madrid: Consejo Superior de Deportes. Centro de Investigaciones Sociológicas.

Heyward, V.H. (2006). Advanced Fitness Assessment and Exercise Prescription, $5^{\mathrm{a}}$ ed. Champaign, IL: Human Kinetics.

Lafont, C., Riviere, D., Vellas, B., López, A. \& Albarede, J.L. (1997). Efectos del ejercicio físico sobre el envejecimiento. Año gerontológico, 11, 199-245.

Lehr, U. (1999). El envejecimiento activo. El papel del individuo y de la sociedad. Revista española de geriatría y gerontología, 34, 314-318.

López, R. \& Rebollo, S. (2002). Análisis de la relación entre práctica deportiva y características sociodemográficas en personas mayores. Revista Internacional de Medicina y Ciencias de la Actividad Física y del Deporte, 5. Online. Available: http://cdeporte.rediris.es/revista/revista5/artcalidad.html [Consultado: Noviembre, 2008].
Matsudo, S. \& Matsudo, V. (1992). Prescription and benefits of physical activity in the third age. Revista Brasileira de Ciencia \& Movimiento, 6, 19-30.

Matsudo, S., Matsudo, V. \& Neto, T. (2000). Efeitos benéficos da actividade física na aptidao física e saúde mental durante o processo de envelhecimento. Revista Brasileira de Actividade Física \& Saúde, 5, 60-76.

Meléndez, A. (2000). Actividades Físicas para los mayores. Las razones para hacer ejercicio. Madrid: Gymnos.

Méndez, A. \& Fernández, J. (2005). Prescripción de la actividad física en personas mayores: recomendaciones actuales. Revista Española de Educación Física y Deportes, 3, 19-29.

Myers, J., Kaykha, A., George, S., Abella, J., Zaheer, N., Lear,S., Yamakazi, T., \& Froelicher, V. (2004). Fitness versus physical activity patterns in predicting mortality in men. American Journal of Medicine, 117, 912-918.

Otero, J.M. (2004). Hábitos y actitudes de los andaluces ante el deporte (2002). Cádiz: Consejería de Turismo y Deporte. Observatorio del Deporte Andaluz.

Paffenbarger, R.S. \& Lee, I.M. (1996). Physical activity and fitness for health and longevity. Research Quarterly for Exercise and Sport, 67 (Supplement), 1128.

Pastor, J.L. (2005). Tercera edad, actividad física y estado de bienestar. Revista Española de Educación Física y Deportes, 3, 13-18.

Portillo, M. (2002). Clásicos de Tai Chi: introducción a la filosofia y a la práctica de una tradición milenaria china. Barcelona: Oniro.

Ruiz, L.M. (1995). El aprendizaje de tareas en las edades avanzadas. En P.L. Rodríguez García \& J.A. Moreno Murcia. Perspectivas de actuación en Educación Física (pp. 153-161). Murcia: Universidad de Murcia.

Schutzer, K.A., \& Graves, B.S. (2004). Barriers and motivations to exercise in older adults. Preventive Medicine: An International Journal Devoted to Practice and Theory, 39, 1056-1061.

Serra, R. \& Bagur, C. (2004). Prescripción de ejercicio fisico para la salud. Barcelona: Paidotribo.

Taylor, A.H., Cable, N.T., Faulkner, G., Hillsdon, M., Narici, M. \& Van Der Bij, A.K. (2004). Physical activity and older adults: a review of health benefits and the effectiveness of interventions. Journal of Sports Sciences, 22, 703-725.

Taylor-Piliae, R. \& Froelicher, E.S. (2004). Effectiveness of Tai Chi exercise in improving aerobic capacity: a meta-analysis. The Journal of Cardiovascular Nursing, 19, 48-57.

Taylor-Piliae, R., Haskell, W.L., Stotts, N.A. \& Froelicher, E.S. (2006). Improvement in balance, strength, and flexibility after 12 weeks of Tai chi exercise in ethnic Chinese adults with cardiovascular disease risk factors. Alternative Therapy in Health and Medicine, 12, 50-58.

Ticó, J. (1995). La actividad física en la ancianidad: actualidad y perspectivas. En P.L. Rodríguez García \& J.A. Moreno Murcia. Perspectivas de actuación en Educación Física (pp. 139-151). Murcia: Universidad de Murcia.

Torrado, V.M., Aparici, M. \& Sanz, P. (1994). Efectos psicológicos de un programa

Torrado, V.M., Aparici, M. \& Sanz, P.(1994). Efectos psicológicos de un programa
de entrenamiento físico en sujetos mayores de 60 años. Revista Española de Geriatría y Gerontología, 29, 138-142.

Tsang, W. \& Hui-Chan, C. (2003). Effects of tai chi on joint proprioception and stability limits in elderly subjects. Medicine and Science in Sports and Exercise, 35, 1962-1971.

Tsang, W. \& Hui-Chan, C. (2004a). Effect of 4- and 8-wk Intensive Tai Chi training on balance control in the elderly. Medicine and Science in Sports and Exercise, 36, 648- 657.

Tsang, W. \& Hui-Chan, C. (2004b). Effects of exercise on joint sense and balance in elderly men: Tai Chi versus golf. Medicine and Science in Sports and Exercise, 36, 658-667.

Tsang, W., Wong, V., Fu, S. \& Hui-Chang, C. (2004). Tai Chi improves standing balance control under reduced or conflicting sensory conditions. Archives of Physical Medicine and Rehabilitation, 85 (1), 129-137

USDHHS (1996). Physical activity and health: A report of the Surgeon General, U.S. Department of Health and Human Services, Public Health Service, Centers for Disease Control and Prevention, National Center for Chronic Disease Prevention and Health Promotion, Division of Nutrition and Physical Activity. Atlanta, GA: USDHHS

USDHHS (U.S. Department of Health and Human Services) (1999). Promotion physical activity: a guide for community action, U.S. Department of Health and Human Services, Public Health Service, Centers for Disease Control and Prevention, National Center for Chronic Disease Prevention and Health Promotion, Division of Nutrition and Physical Activity. Atlanta, GA: USDHHS

WHO(World Health Organization) (1997). The Heidelberg guidelines for promoting physical activity among older persons. Journal of Aging and Physical Activity, $5,1-8$.

Zhang, J.G., Ishikawa-Takata, K., Yamazaki, H., Morita, T. \& Ohta, T. (2006). The effects of Tai Chi Chuan on physiological function and fear of falling in the less robust elderly: an intervention study for preventing falls. Archives of Gerontology and Geriatrics, 42, 107-116. 